Relato de Caso

\title{
Diagnóstico e conduta pré-natal em malformação adenomatóide cística pulmonar fetal: apresentação de um caso
}

\author{
Prenatal diagnosis and therapy for fetal cystic adenomatoid \\ pulmonary malformation: a case report

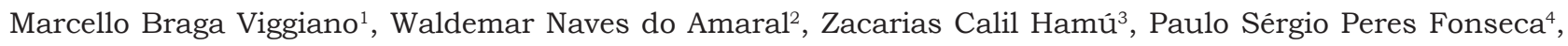 \\ Juliane Damasceno de Castro $^{5}$, Francielle Pulcinelli ${ }^{6}$
}

\section{RESUMO}

A malformação adenomatóide cística fetal consiste em anomalia do desenvolvimento pulmonar, proveniente de crescimento anormal dos bronquíolos respiratórios terminais. Por tratar-se de malformação rara, nem sempre é lembrada como possibilidade diagnóstica. Apresentamos o relato de um caso de malformação adenomatóide cística pulmonar fetal em adolescente primigesta, ressaltando a importância do seu diagnóstico precoce e possibilidades terapêuticas. Apresentamos também o aspecto evidenciado após a colocação de cateter para drenagem contínua.

PALAVRAS-CHAVE: Diagnóstico pré-natal; Feto/anormalidades; Malformação adenomatóide cística congênita do pulmão

\section{ABSTRACT}

Fetal cystic adenomatoid malformation is a pulmonary developmental anomaly arising from an overgrowth of the terminal respiratory bronchioles. This is such a rare malformation, that is not always thought of as a diagnostic possibility. We present a case of pulmonary cystic adenomatoid malformation and emphasize the importance of early diagnosis and therapeutic possibilities. We also present its evolution after prenatal placement of a catheter for continuous drainage.

KEYWORDS: Prenatal diagnosis; Fetus/abnormalities; Cystic adenomatoid malformation of lung, congenital

\section{Introdução}

As alterações císticas envolvendo as estruturas intratorácicas fetais são, em sua maioria, acessíveis à propedêutica ultra-sonográfica durante o periodo intra-uterino.

$\mathrm{Na}$ avaliação do tórax fetal normal, os órgãos identificáveis são os pulmões e o coração, entretanto, em face de situações patológicas, estruturas torácicas invisiveis ao exame normal, tais como, espaço pleural, espaço pericárdico e mediastino, são de fundamental importância para se firmar um diagnóstico provável e conduzir apropriadamente cada situação ${ }^{1}$.

A malformação adenomatóide cística pulmonar (MACP) é caracterizada por anomalia de desenvolvimento do epitélio bronquiolar endodérmico e seu mesênquima adjacente originando a proliferação excessiva das estruturas respiratórias terminais. Esta falha embrionária ocorre entre a $6^{\mathrm{a}}$ e a $8^{\mathrm{a}}$ semana após a concepção, sendo que algumas lesões podem se desenvolver mais tardiamente entre a $14^{\mathrm{a}}$ e a $18^{\mathrm{a}}$ semana da gravidez ${ }^{2}$.

1 Chefe do Serviço de Medicina Fetal do Hospital Materno Infantil de Goiânia/Professor Substituto do Departamento de Ginecologia e Obstetrícia da Faculdade de Medicina da Universidade Federal de Goiás - UFG - Goiânia (GO) - Brasil.

2 Chefe do Departamento de Ginecologia e Obstetrícia da Faculdade de Medicina da Universidade Federal de Goiás - UFG - Goiânia (GO) - Brasil.

3 Chefe do Serviço de Cirurgia Pediátrica do Hospital Materno Infantil de Goiânia (GO) - Brasil.

4 Chefe do Serviço de Patologia do Hospital Materno Infantil de Goiânia (GO) - Brasil.

5 Residente de Ginecologia e Obstetrícia do Hospital Materno Infantil de Goiânia (GO) - Brasil.

6 Acadêmica da Faculdade de Medicina da Universidade Federal de Goiás - UFG - Goiânia (GO) - Brasil.

Correspondência: Marcello Braga Viggiano

Avenida $5^{a}$ Radial, n 79 apt. 202 - Setor Pedro Ludovico - 74823-030 - Goiânia - GO - Telefone: celular (62) 9979-0835; residência (62) 241-0572 e-mail: marcelloviggiano@ig.com.br

Recebido em: 18/11/2004

Aceito com modificações em: 11/4/2005

Rev Bras Ginecol Obstet. 2005; 27(6):353-6 
Sua real incidência é desconhecida e esporádica, não havendo fatores teratogênicos ou anormalidades cromossômicas relacionadas, existindo prevalência de 1,7 recém-nascidos do sexo masculino para cada feminino ${ }^{3}$.

A classificação mais comumente adotada tem como parâmetro o tamanho dos cistos encontrados. O tipo I contém vários cistos volumosos, irregulares ou cisto predominante circundado por menores, medindo em média 2 a $7 \mathrm{~cm}$ de diâmetro; o tipo II apresenta múltiplos pequenos cistos, medindo até $12 \mathrm{~mm}$; e o tipo III é composto por volumosa massa densa, consistente, contendo microcistos ${ }^{4}$.

Em 1985, Adzick et al. ${ }^{5}$ propuseram classificação simplificada, dividindo as MACPs em três grupos: forma macrocística (cistos $>5 \mathrm{~mm}$ de diâmetro), forma microcística (cistos $<5 \mathrm{~mm}$ ) e forma mista.

As anomalias associadas são raras (6-10\%), porém existem casos descritos na literatura que são acompanhados por agenesia renal bilateral, rins multicísticos, displasias renais, hidrocefalia, anomalias cardiacas (tetralogia de Fallot), atresia jejunal ou ileal, deformidades de clavícula e coluna, sirenomelia e hérnia diafragmática ${ }^{6}$.

O objetivo deste trabalho é reforçar que, apesar de pouco comum, a MACP deve ser incluída como possibilidade diagnóstica em face de massas torácicas fetais e ilustrar, de maneira clara, o excelente suporte oferecido pela Medicina Fetal em relação à propedêutica e terapêutica no caso em questão.

\section{Descrição do caso}

Gestante adolescente, 19 anos incompletos, primigesta, foi submetida à ultra-sonografia obstétrica de rotina em serviço externo, que identificou imagens císticas em topografia pulmonar, sendo então encaminhada a serviço de referência em Medicina Fetal, onde foi realizada ultra-sonografia morfológica em 14 de junho de 2004, que identificou gestação única, do sexo masculino, biometricamente e adequadamente compativel com 26 semanas de gestação, apresentando três imagens císticas adjacentes que ocupavam os lobos pulmonares médio e superior do hemitórax direito, de formas arredondadas e volumes variados, anecogênicas, homogêneas e não pulsáteis, medindo os diâmetros da maior imagem cística $2,7 \times 3,7 \mathrm{~cm}$ (Figuras 1 e 2). O volume de líquido amniótico encontrava-se no percentil 85 para a respectiva idade gestacional, sendo ainda visualizado e confirmado por meio de ecocardiografia discreto desvio de mediastino à esquerda, com anatomia e funções valvares cardiacas preservadas. Optou-se en- tão, após consentimento da paciente, por tentativa de derivação pleuro-amniótica com a utilização de cateter adaptado de bloqueio anestésico peridural. O procedimento foi realizado em 30 de junho de 2004 sem intercorrências imediatas ou tardias, na $28^{\mathrm{a}}$ semana de gestação, sendo aspirado $5 \mathrm{~mL}$ de líquido citrino durante a punção, que nos revelou em exame citopatológico escassa quantidade de linfócitos e concentração total protéica de $39 \mathrm{mg} / \mathrm{dL}$. Nas consultas de acompanhamento, observou-se redução gradual nas dimensões císticas, sendo que no primeiro dia após derivação, o maior cisto media $2,3 \times 1,7 \mathrm{~cm}$; no $5^{\circ}$ dia 2,3 x 2,3 cm; e no $12^{\circ}$ dia $1,7 \times 1,9 \mathrm{~cm}$. Houve ainda retorno do mediastino à sua posição normal, sendo que em todos os exames ultra-sonográficos, confirmou-se a correta localização das extremidades do cateter introduzido. A partir da $36^{\mathrm{a}}$ semana de gestação, o volume do cisto permaneceu constante, apesar de confirmada a correta localização do cateter, sugerindo processo obstrutivo de drenagem. O parto deu-se na $38^{a}$ semana, por via abdominal. No dia da interrupção, as dimensões da imagem cística eram 3,0 × 2,9 cm. O recém-nascido pesou 3.070 g e a evolução pós-natal foi normal. Aos 4 dias de vida, optou-se por realização de lobectomia superior pulmonar direita eletiva com achado cirúrgico macroscópico de tumoração cística com conteúdo líquido citrino, cápsula regular e fina, medindo em seu maior diâmetro 3,0 $\mathrm{cm}$, tendo evolução pós-cirúrgica dentro dos padrões de normalidade. O estudo anatomopatológico e os achados histológicos de epitélio colunar e cubóide delineando a cavidade cística associada à presença de células musculares lisas nas paredes dos cistos confirmaram a suspeita de malformação adenomatóide cística pulmonar (Figura 3). Em consulta de acompanhamento aos três meses de vida (7 de dezembro de 2004) o lactente apresentava peso e estatura adequados, além de parâmetros cárdio-respiratórios normais para a idade.

\section{Discussão}

O diagnóstico pré-natal da MACP é sempre ultra-sonográfico, sendo realizado, mais comumente, a partir do segundo trimestre da gestação. A forma mais comum de apresentação ultrasonográfica da MACP é como imagens císticas de tamanhos variáveis, únicas ou múltiplas (mais freqüentes), hipoecogênicas, homogêneas e não pulsáteis. As lesões unilaterais são mais comuns (85\% dos casos), atingindo somente um lobo pulmonar, preferencialmente o inferior ${ }^{1,7}$. 


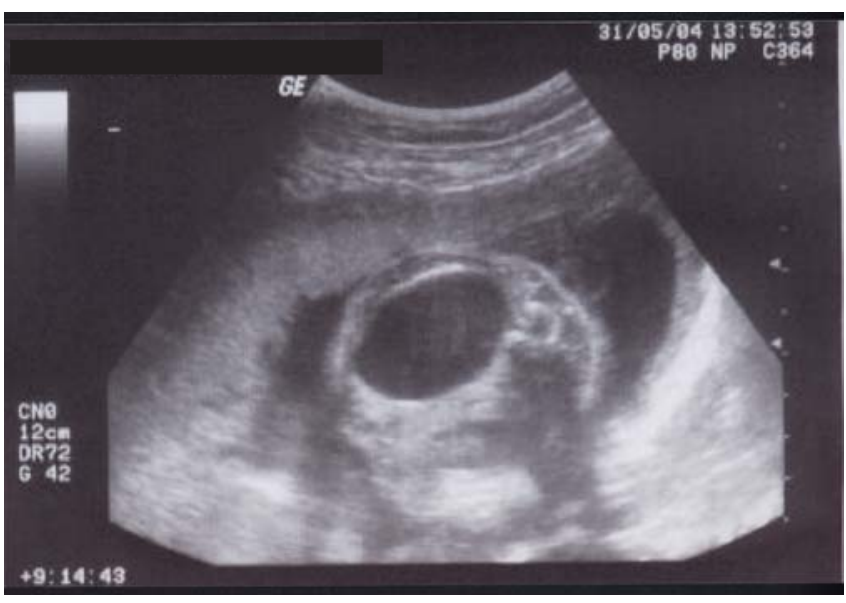

Figura 1 - Forma mais comum de manifestação ultra-sonográfica da malformação adenomatóide cística pulmonar ao modo bidimensional. Imagem circular, anecogênica (cística), homogênea, localizada no hemitórax direito fetal. (Plano transversal do tórax)

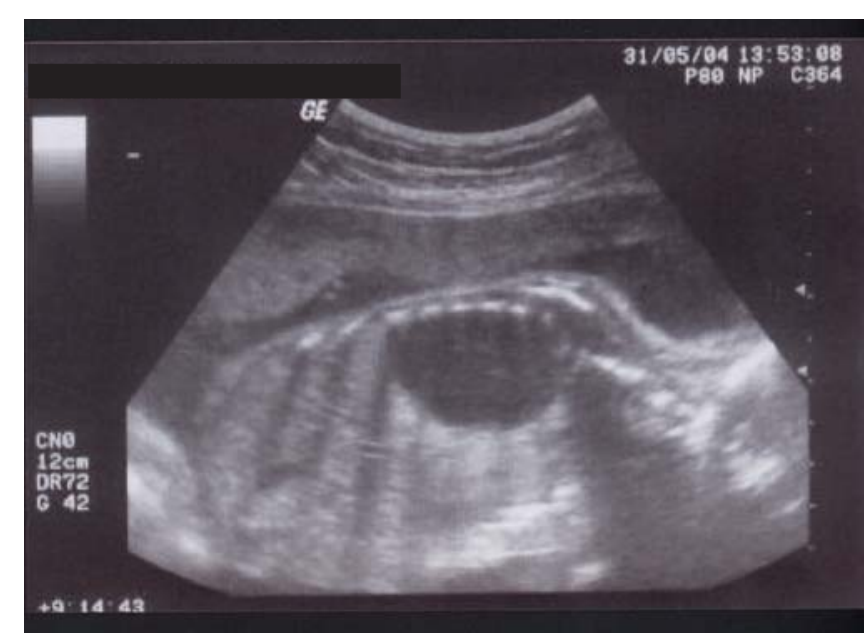

Figura 2 - Imagem ultra-sonográfica bidimensional de malformação adenomatóide cística pulmonar volumosa demonstrando sua localização nos lobos pulmonares médio e superior do hemitórax direito fetal. (Plano longitudinal do tórax)

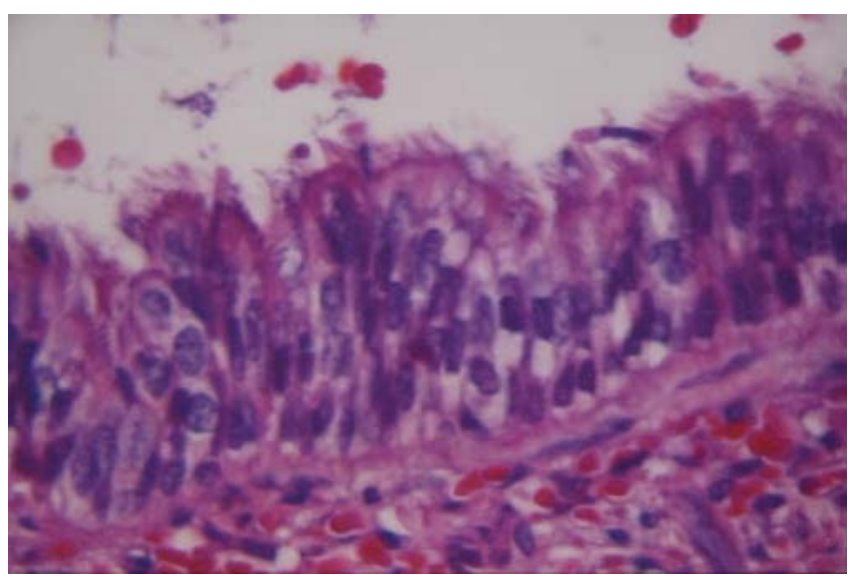

Figura 3 - Achados histológicos de malformação adenomatóide cística pulmonar: epitélio colunar e cubóide delineando a cavidade cística associada à presença de células musculares lisas nas paredes dos cistos.
O estudo com doplervelocimetria colorida auxilia no diagnóstico diferencial, principalmente com o seqüestro pulmonar, e na distinção entre suas formas extralobar e intralobar ${ }^{8}$.

O diagnóstico diferencial das imagens císticas pulmonares é realizado em face das anomalias intratorácicas (malformação adenomatóide cística, seqüestro pulmonar, cisto broncogênico e atresia brônquica) e extratorácicas (hérnia diafragmática), sendo por muitas vezes, o diagnóstico definitivo confirmado somente no período pósnatal. Vale ressaltar que existem relatos de coexistência de seqüestro pulmonar e MACP, e que a primeira pode mimetizar inclusive alguns critérios histológicos de diagnóstico desta última, sugerindo que não existe linha marcante de diferença do ponto de vista embriológico entre as duas condições ${ }^{9}$.

Entre as complicações possiveis destacamse: hipoplasia pulmonar, insuficiência cardiaca devido a desvio do mediastino, derrames cavitários, hidropisia e polidramnia. A fisiopatologia do aumento do volume de líquido amniótico baseia-se na compressão esofageana extrínseca, diminuição da absorção pelo pulmão hipoplásico e ainda cogita-se da produção anormal de líquido pulmonar pelos pneumócitos do tipo $2^{10}$.

A evolução da MACP depende do seu tipo (sendo menos favorável na do tipo III), localização, volume, presença ou não de outras anomalias associadas e suas possiveis complicações, como a hidropisia ${ }^{11}$.

Estes fenômenos levam à instalação de quadro de hidropisia fetal com derrame pleural, ascite e edema de subcutâneo, dependente da gravidade (tamanho da lesão) e o tempo de instalação do quadro. A presença ou não de hidropisia muda o prognóstico e a conduta pré-natal. Sendo assim, sinais precoces de insuficiência cardiaca devem ser pesquisados à ultra-sonografia e acompanhados mesmo nos casos de pequena manifestação e estáveis ${ }^{1}$.

A gravidez deverá ser monitorada com ultrasonografias seriadas a fim de acompanhar o crescimento ou a involução da malformação e detectar o surgimento de possiveis complicações. Para aqueles casos em que já se instalou a hidropisia fetal ou derrame pleural em idade gestacional menor que 32 semanas, deve ser considerada a indicação de derivação tóraco-amniótica. Nos casos de compressão mediastinal por grandes massas contendo fartas porções císticas, há que se considerar a punção torácica para seu esvaziamento e, se possivel, derivação contínua por meio da colocação do cateter ${ }^{11}$. 
A conduta nos casos de anomalias císticas intratorácicas ainda não é consensual na literatura científica, variando desde expectante até a realização de procedimentos invasivos, como punções seriadas ou derivações definitivas ${ }^{12}$.

A importância do diagnóstico pré-natal das malformações císticas intratorácicas reside no fato de estas serem responsáveis, ao nascimento, por quadros de insuficiência respiratória grave em conseqüência à hipoplasia pulmonar e insuficiência cardíaca devida a desvio do mediastino. A identificação e seguimento adequado destas anomalias, em serviço de Medicina Fetal, tornam-se fundamentais para a programação de assistência neonatal especializada.

\section{Referências}

1. Isfer EV, Chrisóstomo AP, Pastore AR. Malformações fetais torácicas. In: Cerri GG, editor. Ultra-sonografia em ginecologia e obstetrícia. Rio de Janeiro: Revinter; 2003. p.230-8.

2. Vergnes P, Chateil JF, Boissinot F, Galperine RI, Demarquez JL, Vital C, et al. Malformations pulmonaires de diagnostic anténatal. Chir Pediatr. 1999;30(4):185-92.

3. Akrivis C, Varras M, Demou A, Bellou A, Stefanaki $\mathrm{S}$, Antoniou N. Prenatal diagnosis of congenital cystic adenomatoid lung malformation: case report and review of the literature. Clin Exp Obstet Gynecol. 2003;30(4):259-62.

4. Donn SM, Martin JN Jr, White SJ. Antenatal ultrasound findings in cystic adenomatoid malformation. Pediatr Radiol. 1981;10(3):180-2.
5. Adzick NS, Harrison MR, Glick PL, Golbus MS, Anderson RL, Mahony BS, et al. Fetal cystic adenomatoid malformation: prenatal diagnosis and natural history. J Pediatr Surg. 1985;20(5):483-8.

6. Ankermann T, Oppermann HC, Engler S, Leuschner I, Von Kaisenberg CS. Congenital masses of the lung, cystic adenomatoid malformation versus congenital lobar emphysema: prenatal diagnosis and implications for postnatal treatment. J Ultrasound Med. 2004;23(10):1379-84.

7. Cohen RA, Moskowitz PS, McCallum WD. Sonographic diagnosis of cystic adenomatoid malformation inutero. Prenat Diagn. 1983;3(2):139-43.

8. De Nicola ALA, Frigério MV, Zanforlin Filho SM, Gollop TR. Diagnóstico pré-natal de seqüestração pulmonar: apresentação de um caso. Rev Bras Ginecol Obstet. 2003;25(3):207-10.

9. Chandran H, Upadhyay V, Pease PW. Congenital cystic adenomatoid malformation and extralobar sequestration occurring independently in the ipsilateral hemithorax. Pediatr Surg Int. 2000;16(12):102-3.

10. Usui N, Kamata S, Sawai T, Kamiyama M, Okuyama H, Kubota A, et al. Outcome predictors for infants with cystic lung disease. J Pediatr Surg. 2004;39(4):603-6.

11. Pumberger W, Hormann M, Deutinger J, Bernaschek G, Bistricky E, Horcher E. Longitudinal observation of antenatally detected congenital lung malformations (CLM): natural history, clinical outcome and long-term follow-up. Eur J Cardiothorac Surg. 2003;24(5):703-11.

12. Morikawa M, Yamada H, Okuyama K, Hirayama Kato E, Watari M, Kataoka S, et al. Prenatal diagnosis and fetal therapy of congenital cystic adenomatoid malformation type I of the lung: a report of five cases. Congenit Anom (Kyoto). 2003;43(1):72-8. 